Opinión

\section{PREVENCIÓN DEL RIESGO EN SANTO DOMINGO DE HEREDIA, COSTA RICA: REUBICACIÓN DEL BARRIO FÁTIMA}

\author{
Mario Fernández Arce $^{1^{*}}$, Carlos Ramírez Umaña ${ }^{2}$ y Rudy Muñoz Jiménez ${ }^{3}$
}

Este artículo de opinión aborda el proceso de reubicación del Barrio Fátima de Santo Domingo de Heredia, un vecindario que había sido inundado y amenazado por deslizamientos en el pasado y declarado inhabitable y reubicable. Reubicar asentamientos humanos es difícil y algunos gestores de riesgo consideran tal medida una utopía. Por lo anterior, es de suma importancia documentar el caso y generar información para una futura plataforma sobre Gestión de Riesgo del cantón. La divulgación de esta medida podría motivar a otras entidades a ejecutar acciones similares en sus territorios, lo cual es una meta del Plan Nacional de Gestión de Riesgos en Costa Rica. El artículo se basó en investigación bibliográfica y estudio de campo. El resultado más importante es que se documentó un caso de reducción (prevención) del riesgo en Costa Rica, lo cual muestra cómo es posible reubicar comunidades expuestas a una o varias amenazas.

\section{INTRODUCCIÓN}

Este trabajo es la documentación del proceso de reubicación del Barrio Fátima de Santo Domingo de Heredia (Figura 1), una acción de prevención de emergencias y desastres. El mismo consta de una descripción de los principales impactos de las inundaciones en el barrio, una cuantificación de las viviendas afectadas y los pasos seguidos hasta reubicar a la comunidad en cuestión. También se incluye una breve descripción de lo que se hará en la zona desalojada en el futuro. Barrio Fátima se encuentra al noroeste de la ciudad de Santo Domingo, muy cerca del límite entre los cantones Santo Domingo y San Pablo

Las medidas preventivas en el contexto de la gestión del riesgo aparte de ser escasas no siempre se documentan y por eso, no quedan registradas en las bases de datos o plataformas de información sobre riesgos y desastres. En el cantón de Santo Domingo hay interés en implementar un sistema de información para la gestión de riesgos y en él habría que incluir todas las actividades preventivas que se han hecho en el cantón. La iniciativa de reubicar Barrio Fátima no había sido documentada y por tanto, la valiosa información alrededor de ella no ha estado disponible para incluirla en una eventual plataforma de información sobre gestión de riesgo del cantón.

Es por lo anterior que se hizo la documentación. La sociedad merece conocer esa buena práctica. Al divulgar esta iniciativa se pretende motivar a los gestores de riesgo para que fomenten la reducción del riesgo mediante acciones preventivas como el desalojo de zonas de alto riesgo, lo cual es una meta del Plan Nacional de Gestión de Riesgos (CNE, 2016). Esta documentación crea información oportuna, concisa y precisa que podría ser incorporada en una futura plataforma de información sobre riesgos y desastres para Santo Domingo.
1. Escuela de Geografía, Universidad de Costa Rica, San José, Costa Rica.

2. Servicio Geológico Ambiental SeGeoAm, San José, Costa Rica.

3. Consultor independiente, San José, Costar Rica.

*Autor de correspondencia: mario.fernandezarce@ucr. ac.cr

\section{RECIBIDO}

11 de marzo de 2020

\section{PUBLICADO}

1 de julio de 2020

\section{Formato cita}

Recomendada (APA): Fernández Arce, M., Ramírez Umaña, C. y Muñoz Jiménez, R. (2020). Prevención del Riesgo en Santo Domingo de Heredia, Costa Rica: Reubicación del barrio Fátima. Revista de Estudios Latinoamericanos sobre Reducción del Riesgo de Desastres REDER, 4(2), 126-133.

\section{() $(\Phi \otimes$}

Todos los artículos publicados en REDER siguen una política de Acceso Abierto y se respaldan en una Licencia CreativeCommons Atribución-NoComercial 4.0 Internacional.

\section{Revista de Estudios} Latinoamericanos sobre Reducción del Riesgo de Desastres (REDER)

Diseño: Lupe Bezzina Tipografía: Hospital 


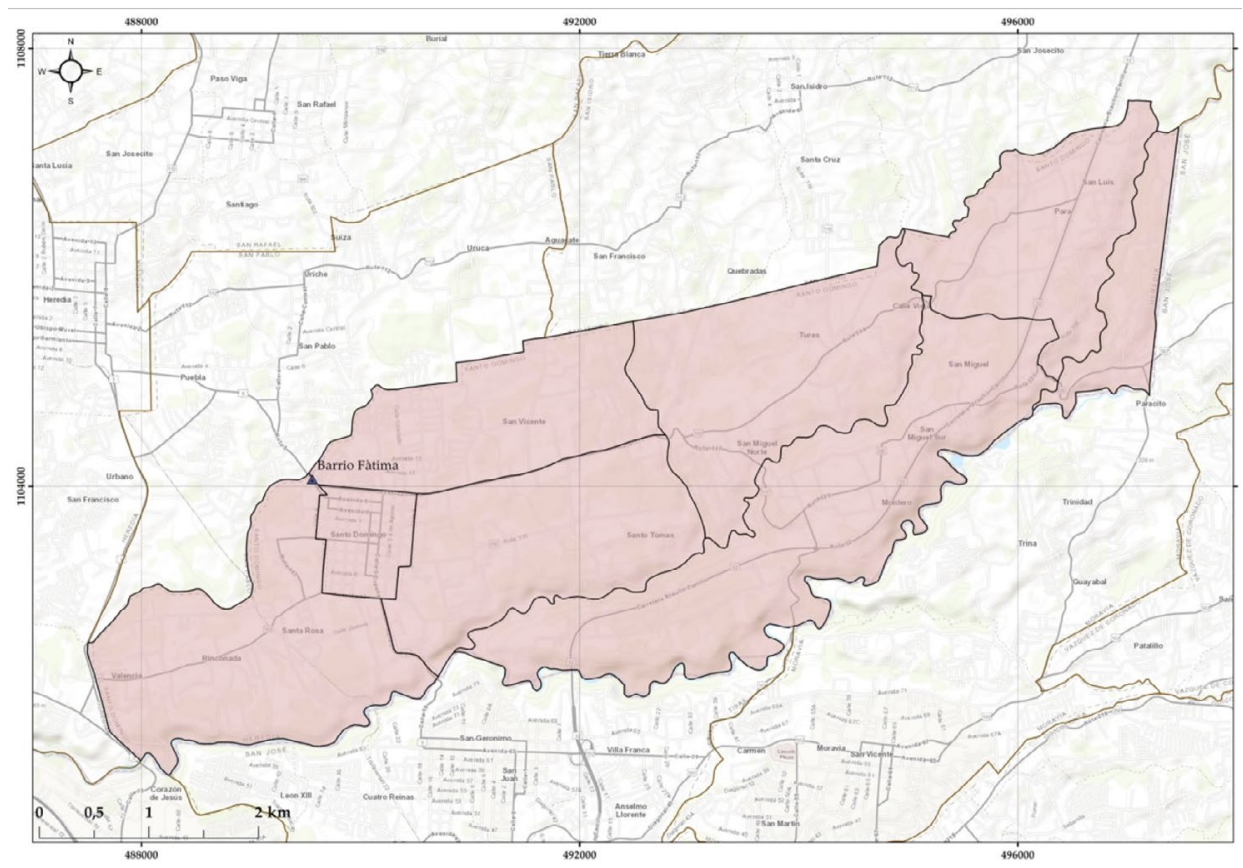

Figura 1. Ubicación de la zona de estudio Fuente: Autores, 2020

\section{METOdOLOGÍA}

Este estudio requirió la revisión de la información sobre las inundaciones y su manejo en la zona de estudio. Ello implicó buscar, seleccionar, analizar y recuperar datos de los incidentes ocurridos en el lugar y del manejo que se hizo para enfrenar la situación. Entre los escritos sobre el tema interesaban informes sobre emergencias pasadas y la información específica sobre viviendas afectadas y declaradas inhabitables. Hubo que identificar en los reportes los textos de interés y tenerlos a disposición para la posterior extracción de los contenidos. Una vez localizados los datos que se necesitaba se hizo una descripción física de los documentos útiles y de los contenidos para clasificarlos y hacer un resumen analítico para facilitar el acceso en otras ocasiones. Se revisaron los documentos para encontrar la información deseada o de interés, la que satisfacía las necesidades de los investigadores. En este paso fue posible identificar los diversos subtemas de interés del estudio como los sitios a trasladar y planes a futuro en el terreno abandonado.

Una segunda opción para obtener la información fue la entrevista a líderes y lideresas del cantón y a miembros del barrio afectado. Las entrevistas fueron personales y abiertas, eran conversatorios para conocer tanto los planes de reubicación como la situación psicosocial de las personas afectadas.

Se hizo trabajo de campo que incluyó visitas a Barrio Fátima para conocer, mediante observación directa, el grado de exposición de las viviendas y sus residentes a las inundaciones y los deslizamientos. Aprovechando tales expediciones, se impartieron charlas a los miembros del barrio sobre las amenazas que los acechaban, con miras a prepararlos para enfrentar y motivarlos para conformar un comité comunal de emergencias. De particular interés fue escuchar el testimonio de personas sobre sus vivencias y luchas contra los eventos naturales y sobre su apego al lugar, independientemente del peligro. También se visitó la urbanización La Zamora, uno de los dos destinos de los reubicados. Este se conoció desde que era un asentamiento informal y se observó su desarrollo hasta convertirse en una urbanización de bien social. Se verificó que en este nuevo habitad los vecinos de Barrio Fátima iban a tener un buen lugar para vivir, el cual tenía casas con patio y hasta una planta para el tratamiento de aguas residuales. Se tomaron notas de las conversaciones con los vecinos y fotografías de los paisajes y elementos del espacio que fueron usados para reforzar los argumentos, criterios y conclusiones de esta publicación.

A fin de documentar el traslado, se hizo una nueva visita a Barrio Fátima una vez ejecutado el traslado de las familias con el fin de tomar fotografías desde un Vehículo Aéreo No Tripulado (VANT). Tales productos sirvieron para mostrar la demolición de las viviendas y el fin de un 
vecindario que por muchos años convivió con la probabilidad de pérdidas materiales y de vidas humanas, a causa de las inundaciones.

\section{RESULTADOS}

\section{Las inundaciones}

Las inundaciones en Barrio Fátima son conocidas desde finales de los años sesenta e inicios de los setenta pero por ese tiempo no eran tan destructivas como en la actualidad. De acuerdo con Fernández et al. (2013), antes de 1999 el lugar no fue afectado tan severamente por desbordamientos de ríos. Pero la situación cambió significativamente a finales de la década de los 90 por la disminución de las zonas permeables en los suelos y, consecuentemente, por el aumento de la impermeabilización de los mismos. Lo anterior responde a la expansión del proceso de urbanización que, por la carencia de suelos aptos, ha llegado hasta las laderas y zonas de inundación de los ríos y quebradas urbanas. Santo Domingo es un cantón más rural que urbano en el que, al igual que en los cantones vecinos, habían excelentes cultivos de café hace unas décadas. Pero dichos cultivos ya no existen porque en los últimos años se ha dado un gran desarrollo de urbanizaciones, tanto en dicho cantón como en los territorios vecinos, que han sustituido por completo tales plantaciones y que han llevado a la impermeabilización de lo suelos y al incremento de la escorrentía superficial. El agua que antes se infiltraba ahora va a los desagues de las calles y de ahí a las quebradas y ríos. De manera que, un río otrora insignificante ahora es un gran flujo de agua después de torrenciales aguaceros o durante eventos meteorológicos de varios días. A eso se suma el hecho de que la población y las viviendas han aumentado por lo que también llega más agua a los cauces del cantón. Fernández et al. (2013) indicaron que la impermeabilización de la microcuenca del río Bermúdez pasó del $26 \%$ en 1975 a 53\% en el 2005, lo cual es una razón de peso para el incremento de las inundaciones.

Eventos de gran impacto en Santo Domingo fueron las inundaciones provocadas por el río Bermúdez en 1999, 2007 y 2010. En 1999 ocurrió la primera inundación significativa y notoria, la cual afectó 35 familias, 19 de las cuales recibieron orden de reubicación (Esquivel, 1999; MS, 1999; Bolaños, 2010). En el 2007, un nuevo evento obligó a varias familias a salir y refugiarse en un albergue temporal y por segunda vez se dio a los afectados la orden de abandonar el lugar.

El 20 de julio de 2010 otra inundación afectó el barrio y de nuevo, hubo que restringir la entrada al mismo (Madrigal, 2010) para hacer evaluaciones, valoraciones, estimación de daños y recomendaciones a las familias. Nuevas familias vivían en Barrio Fátima en ese año, muy probablemente grupos familiares que ocuparon las casas dejadas por otros. En el informe de situación que elaboró el Comité Municipal de Emergencias (CME, 2010) se indicó que valoraron 25 viviendas de Barrio Fátima, 22 de las cuales fueron afectadas y por ello se les comunicó a las familias residentes que debían ser reubicadas (CME, 2010). Estos desbordamientos causaron grandes destrozos en Barrio Fátima y dejaron decenas de casas inhabitables.

\section{Declaratorias de reubicación e inhabitabilidad}

Los riesgos para los habitantes de Barrio Fátima eran evidentes desde hace varias décadas y las primeras sugerencias de reubicación del asentamiento se remontan a la década de los 80 . En nota del 6 de octubre de 1987 de Xinia Carvajal y José Luis Vargas Mejía del Ministerio de Salud (MS), dirigida al Departamento de Ingeniería Sanitaria (DIS) del MS, se indica que si el DIS considera que las familias del Vaticano (hoy Barrio Fátima) deben ser evacuadas, la municipalidad consideraría la posibilidad de ubicar las familias en otro sector. Este barrio fue declarado inhabitable y reubicable desde 1991 por problemas de infraestructura, falta de previsión urbanística y cercanía al río Bermúdez. Según el Oficio SC-630-91, las razones iniciales para ello fueron las siguientes: distribución urbana desordenada y sin planificación, letrinas en mal estado, evacuación de aguas servidas por canales abiertos o tuberías improvisadas, falta de acceso a vehículo recolector de residuos sólidos (solo había una canasta única sobre una acequia a la que caían los desechos), carencia de redes de evacuación de aguas pluviales (a raíz de esto habían problemas sanitarios) y falta de condiciones para mejorar. En el oficio SRCN-679-91, del 20 de noviembre de -1991 se informa a Dra. Xinia Carvajal del Centro de Salud de Santo Domingo sobre edificaciones que fueron declaradas inhabitables en diciembre de 1990 en el Vaticano; el informe es suscrito por la Dra. Virginia Céspedes y Sergio Acosta del MS.

En el 2007, con motivo de las inundaciones de ese año en el barrio, en un informe del MS (Arrieta et al., 2007) sobre visita al lugar afectado, se reitera las condiciones de inhabitabilidad 
dadas por condiciones físico-sanitaria deplorables, casas construidas sobre pilotes de madera y desagües directo al río. En dicho informe se indica que han proliferado las viviendas construidas sin la mínima regulación e improvisadas y que hay explotación al inquilinato, hacinamiento y criaderos de Aedes Aegypti. En el oficio ALM 0260-2007 el alcalde Raúl Isidro Bolaños, coordinador de la Comisión Local de Emergencias de Santo Domingo solicitó una reunión urgente al ministro de la vivienda de aquel entonces, Fernando Zumbado, para definir acciones a seguir con el fin de evitar un problema mayor en Fátima, que podría incluir pérdidas de vidas. En el oficio JAOB-786-07 el diputado de la provincia de Heredia José Ángel Ocampo también solicitó una reunión a Zumbado para el mismo fin. Al parecer no hubo respuesta ni solución.

En un informe sobre una Inspección por derrumbes y erosión lateral del río Bermúdez Chaves (2008) reiteró: "Se debe proceder a realizar la reubicación de las familias afectadas" y solicitar a funcionarios del Instituto Mixto de Ayuda Social (IMAS) y Ministerio de Vivienda y Asentamientos humanos (MIVAH) valorar la posibilidad de colaborar para tal reubicación. Herra (2010) indicó: "En las zonas de protección del río Bermúdez no es apto para uso habitacional de ninguna índole". En este informe se recomendó demolición de las viviendas inhabitables, eliminar servicios básicos, prohibir uso habitacional y cumplir con los retiros (de las viviendas con respecto al cauce del río).

La investigación revela que Barrio Fátima ha requerido reubicación desde hace muchos años y por las condiciones del vecindario antes que por las inundaciones. Acciones recomendadas para el asentamiento se muestran en la Tabla 1.

\begin{tabular}{|l|l|l|}
\hline Año & \multicolumn{1}{|c|}{ Causa } & \multicolumn{1}{c|}{ Acción recomendada } \\
\hline 1987 & Problemática detectada & Valoración de las condiciones de las viviendas de Barrio Fátima \\
\hline 1991 & Inadecuadas condiciones del barrio & Recomendar la reubicación del asentamiento \\
\hline 1996 & Denuncia & Trasladar el caso a la Comisión Nacional de Emergencias \\
\hline 1999 & Inundación & Recomendación de reubicación de 19 viviendas \\
\hline 2007 & Inundación & $\begin{array}{l}\text { Suspender los trabajos de mejoramiento de la comunidad. Se } \\
\text { recomendó reubicación de viviendas afectadas. }\end{array}$ \\
\hline 2010 & Inundación & Reubicación del barrio \\
\hline
\end{tabular}

Tabla 1. Cronología de acciones recomendadas para Barrio Fátima Fuente: Ministerio de Salud (2010)

A pesar de la declaratoria de inhabitabilidad de las casas en 1999 no se hizo la reubicación de las familias y en el 2010 las aguas anegaron nuevamente el barrio, causando serios daños y pérdidas materiales (Reyes et al., 2014). Fue a raíz del evento del 2010 que se consideró la gran vulnerabilidad física de los habitantes del lugar y se determinó que el mismo era una zona de alto riesgo por inundación.

\section{Reubicación}

Después del incidente del 2010 varias familias se fueron del barrio, algunas alquilaron casas en otros sitios y otras se fueron donde sus familiares, comenzando así el proceso de reubicación del barrio. La Comisión Especial de Vivienda de la Municipalidad de Santo Domingo inició trámites para trasladar familias a sitios seguros basada en la lista de familias víctimas de la emergencia ocurrida en el 2010. Dicha entidad se apoyó en estudios sobre la condición socio-económica de las familias afectadas y en análisis rigurosos que permitieran saber si los afectados calificaban para asignarles un bono de vivienda en otro sector. El análisis incluía características de la vivienda (propiedad o alquilada), solvencia económica y si era miembro primario o secundario del núcleo familiar. En el proceso de selección también se incluyó la orden sanitaria del Ministerio de Salud en la que se declaró que son casas inhabitables.

Gracias a tales estudios fue posible reubicar familias en las urbanizaciones La Zamora del distrito Tures y Real Dante del distrito Santa Rosa (Figura 2).

En el proyecto de vivienda Real Dante se ubicaron familias de Barrio Fátima que no habían tenido solución de vivienda y estaban identificados en la lista de la Comisión de Emergencias (CEVMSD, 2018).

Es importante indicar que los procesos de selección fueron descalificadas algunas familias por datos errados en la información que ofrecieron, no entregar información requerida, tener solvencia económica o por ser miembro secundario del grupo familiar, todo lo cual consta en el acta antes mencionada. Un dato preocupante es que unas pocas familias se han negado a salir del lugar. 


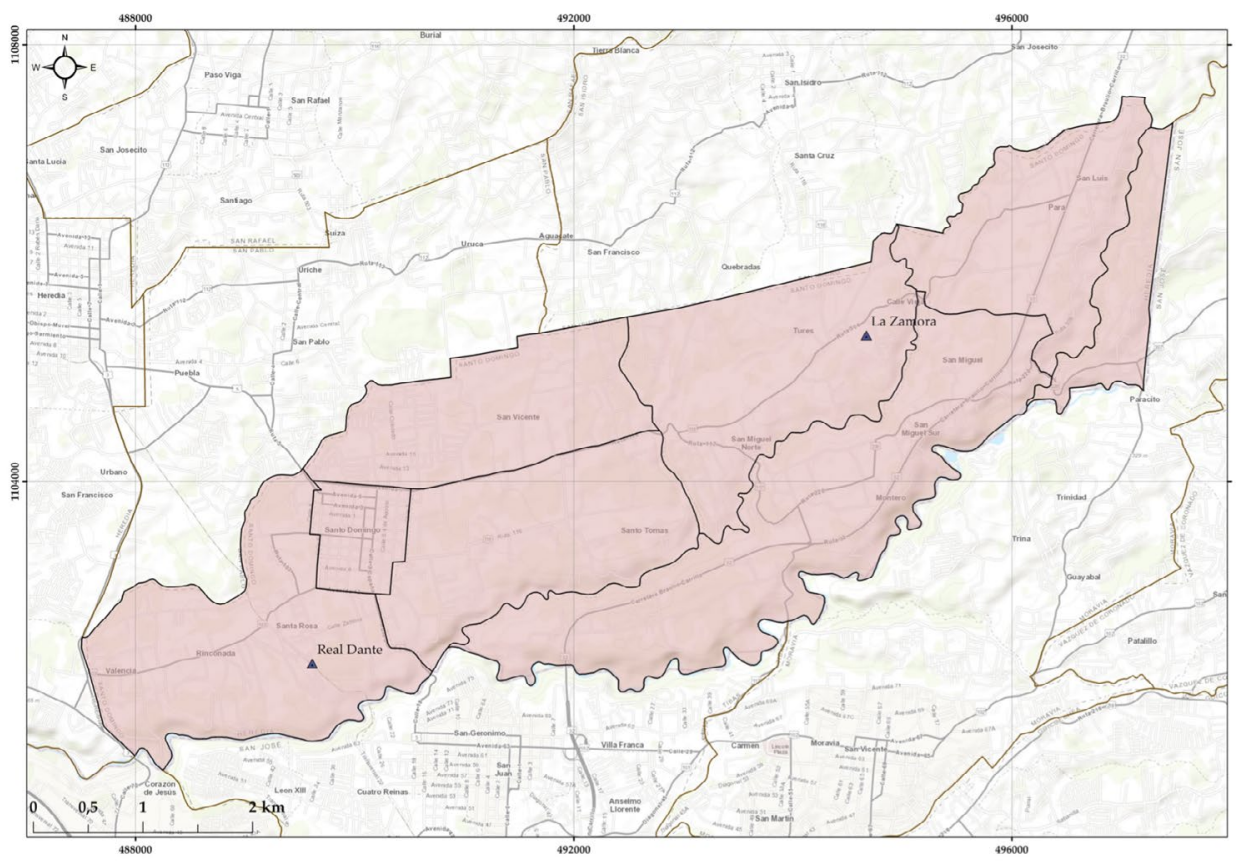

Figura 2. Mapa de las urbanizaciones receptoras de familias Fuente: Autores, 2020

En el primer semestre de 2019 se ejecutó la demolición de las viviendas cuyos dueños ya habían sido trasladados a las urbanizaciones antes mencionadas. No solo se demolieron las casas sino que también se removieron los escombros del lugar como se muestra en la Figura 3, en la cual se aprecia el sitio tal y como quedó después de la limpieza. Se puede apreciar el río en la esquina inferior izquierda de la Figura 3.

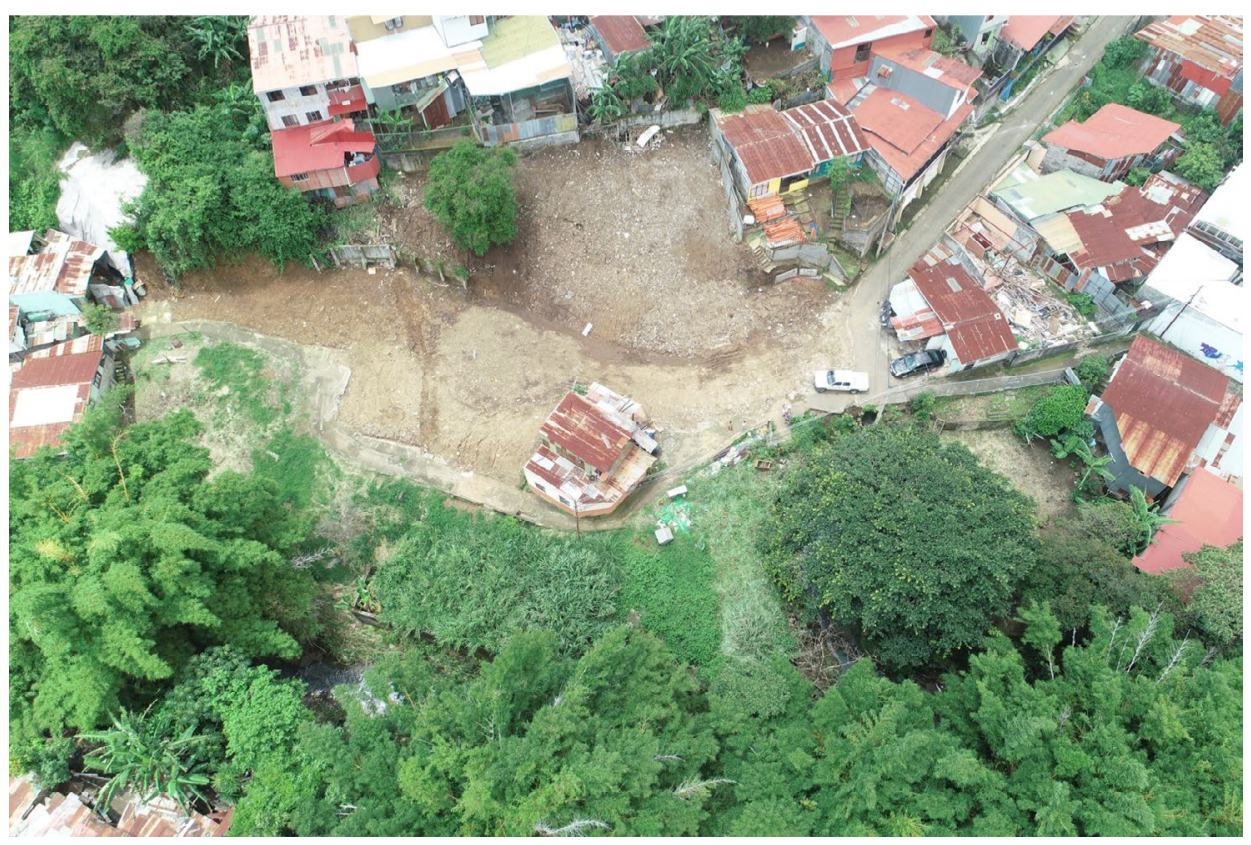

Figura 3. Fotografía aérea de Barrio Fátima

Fuente: Carlos Ramírez Umaña, 2019

\section{DISCUSIÓN}

Los riesgos para los habitantes de Barrio Fátima eran evidentes desde hace varias décadas y las primeras sugerencias de reubicación del asentamiento se remontan a los años 80. En nota del 6 de octubre de 1987 de Xinia Carvajal y José Luis Vargas Mejía del Ministerio de Salud (MS), dirigida al Departamento de Ingeniería Sanitaria (DIS) del MS, se indica que si el DIS considera que 
las familias del Vaticano (hoy Barrio Fátima) deben ser evacuadas, la municipalidad consideraría la posibilidad de ubicar las familias en otro sector. Este barrio fue declarado inhabitable y reubicable desde 1991 por problemas de infraestructura, falta de previsión urbanística y cercanía al río Bermúdez. Según el Oficio SC-630-91, las razones iniciales para ello fueron las siguientes: distribución urbana desordenada y sin planificación, letrinas en mal estado, evacuación de aguas servidas por canales abiertos o tuberías improvisadas, falta de acceso a vehículo recolector de residuos sólidos (solo había una canasta única sobre una acequia a la que caían los desechos), carencia de redes de evacuación de aguas pluviales (a raíz de esto habían problemas sanitarios) y falta de condiciones para mejorar. En el oficio SRCN-679-91, del 20 de noviembre de -1991 se informa a Dra. Xinia Carvajal del Centro de Salud de Santo Domingo sobre edificaciones que fueron declaradas inhabitables en diciembre de 1990 en el Vaticano; el informe es suscrito por la Dra. Virginia Céspedes y Sergio Acosta del MS.

De acuerdo con Fernández et al. (2013), antes de 1999 el lugar no había sido afectado tan severamente por desbordamientos del Río Bermúdez. En 1999 ocurrió la primera inundación significativa y notoria, la cual afectó decenas de familias varias de las cuales recibieron orden de reubicación (CNE, 1999; MS, 1999; MSD, 2010).

En el 2007, con motivo de las inundaciones de ese año en el barrio, en un informe del (MS, 2007) sobre visita al lugar afectado, se reitera las condiciones de inhabitabilidad dadas por condiciones físico-sanitaria deplorables, casas construidas sobre pilotes de madera y desagües directo al río. En dicho informe se indica que han proliferado las viviendas construidas sin la mínima regulación e improvisadas y que hay explotación al inquilinato, hacinamiento y criaderos de Aedes Aegypti. En el oficio ALM 0260-2007 el alcalde Raúl Isidro Bolaños, coordinador de la Comisión Local de Emergencias de Santo Domingo solicitó una reunión urgente al ministro de la vivienda de aquel entonces, Fernando Zumbado, para definir acciones a seguir con el fin de evitar un problema mayor en Fátima, que podría incluir pérdidas de vidas. En el oficio JAOB-786-07 el diputado de la provincia de Heredia José Ángel Ocampo también solicitó una reunión a Zumbado para el mismo fin. Al parecer no hubo respuesta ni solución.

En un informe sobre una Inspección por derrumbes y erosión lateral del río Bermúdez Ignacio Chaves reiteró "Se debe proceder a realizar la reubicación de las familias afectadas" y solicitar a funcionarios del IMAS y MIVAH valorar la posibilidad de colaborar para tal reubicación. Herra (2010) indicó: "En las zonas de protección del río Bermúdez no es apto para uso habitacional de ninguna índole" (CNE, 2008). En este informe se recomendó demolición de las viviendas inhabitables, eliminar servicios básicos, prohibir uso habitacional y cumplir con los retiros (de las viviendas con respecto al cauce del río).

El 20 de julio del 2010, otra inundación afectó el barrio y de nuevo, hubo que restringir la entrada al mismo (DPSD, 2010) para hacer evaluaciones, valoraciones, estimación de daños y recomendaciones a las familias. En el informe de situación que elaboró el Comité Municipal de Emergencias (CMEMSD, 2010) se indicó que valoraron 25 viviendas de Barrio Fátima, 22 de las cuales fueron afectadas y por ello se les comunicó a las familias residentes que debían ser reubicadas (CMEMSD, 2010).

En nota del 10 de febrero de 2012 del Ing. Randall Madrigal, vicealcalde de Santo Domingo, a Patricia Guzmán, Mónica Hoffmaistor, Gerardo Morales y Daniel Vargas, funcionarios municipales, sobre la solicitud de habilitación de vivienda con declaratoria de inhabitabilidad, se giraron instrucciones para denegar la conexión del servicio de electricidad y la rehabilitación de la misma. Asimismo, el Ing. Randall Madrigal indicó a las vecinas de Barrio Fátima Daysi Alvarado y Emilia Alvarado, quienes pretendían construir en sus terrenos, que no se podía dar permisos para ningún tipo de construcción en sus propiedades puesto que están en una zona declarada de alto riesgo (Oficio: DSOT-EXTERNO-040-04 ramal). Esta acciones confirmaron que ya había una seria y firme iniciativa para abandonar Barrio Fátima. Con la inauguración de la urbanización La Zamora en el 2014 y la asignación de viviendas en Real Dante desde 2018 fue posible trasladar familias de Barrio Fátima a tales residenciales. En enero de 2019 se hizo la demolición de las viviendas desalojadas de dicho barrio y con eso se hizo realidad la reubicación de este vecindario domingueño.

De las entrevistas realizadas a las personas afectadas por las inundaciones se determinó que después del evento del 2010 varias familias de Barrio Fátima abandonaron el lugar por su propios medios. Pero los que no tenían otra alternativa permanecieron allí, soportando el temor 
a una nueva inundación en cada época lluviosa y después de cada aguacero torrencial. Algunos afectados expresaron miedo a deslizamientos de tierra que afectaran sus viviendas. Otros datos suministrados dieron cuenta de oposiciones a salir de la zona de riesgo y del incumplimiento de requisitos para optar por vivienda. Miembros de la Comisión Especial de Vivienda de la Municipalidad de Santo Domingo y autoridades locales indicaron que la asignación de residencias se apegó a estudios técnicos y a un riguroso proceso de calificación.

El trámite no fue fácil. No todos salieron y no todos quisieron salir. Es importante reconocer que al menos una familia se negó a salir por apego al terruño (barrio) y por no tener certeza de que la nueva localidad y habitación cumpliera con sus expectativas. Como se indicó previamente, el proceso de selección y calificación de la familias para obtener una vivienda fue difícil. Se encontró familias a las que no se les podía otorgar una casa porque tenían suficientes recursos como para solventar el problema sin la ayuda del Estado o del gobierno local. Esos son aspectos negativos de la reubicación que revelan la dificultad para ejecutar desalojos aun cuando ellos promueven el bienestar y la seguridad de los vulnerables.

Para algunos autores las reubicaciones generan una trasformación negativa y reducen tan solo parcial y temporalmente los riesgos (Briones, 2010). Dicho autor plantea como hipótesis que los modelos de reubicaciones no corresponden a las necesidades socioeconómicas ni a los patrones culturales de las comunidades y que son reveladores de un proceso de traslado y distribución del riesgo. Por su parte, Macías (2008) menciona el desarraigo, alienación de los territorios de pertenencia, estres de reubicación, manipulación de información e implicaciones sociales, políticas y económicas como problemas asociados a las reubicaciones. Este autor indica que, según los expertos, los desplazamientos por impactos de amenazas debe ser una medida de última instancia.

Pese a lo anterior, la acción preventiva en Barrio Fátima se considera exitosa porque resolvió el problema de vivienda a personas que vivían en humildes casas ubicadas en la ladera y llanura de inundación del Río Bermúdez. La condición social y económica de quienes vivían allí eran precarias por lo que una nueva vivienda mejoró su situación. En dicho éxito influyó el hecho de que se trató de un desplazamiento de familias de una pequeña parte de la ciudad de Santo Domingo de Heredia, no de toda una comunidad. No hubo traslado del riesgo por cuanto los destinos de los reubicados son planos y sin ríos cercanos ni otras amenaza que los pueda impactar. Se estima que no hubo gran impacto en las condiciones sociales, económica y sociales de los trasladados.

A manera de conclusión, diremos que la reubicación de asentamientos humanos en zonas de alto riesgo es difícil pero posible y el Barrio Fátima de Santo Domingo de Heredia es un ejemplo de ello. Para llevarla a cabo se requirió un gran esfuerzo tanto de las autoridades a cargo del manejo de los riesgos como de los mismos vulnerables y afectados. También fue necesario un estudio serio y riguroso para determinar el número de familias que califican para recibir solución de vivienda en otro sitio.

De las familias afectadas por las inundaciones y desalojadas en el 2010 se les ha resuelto la situación de vivienda a la mayoría de ellas, unas fueron alojadas en el residencial La Zamora, en el distrito de Tures, y otras en la urbanización Real Dante de Santa Rosa. La reubicación ha mejorado las condiciones de vida de los beneficiarios y ha contribuido positivamente en la liberación de presión sobre suelo urbano cercano a cauce del Río Bermúdez. Las viviendas de las familias trasladadas fueron totalmente demolidas y el sitio que ocupaban está completamente limpio y libre de escombro, esperando ser protegido por una barrera de alambre y convertido en una zona de bosque. El plan futuro podría ser acondicionar el lugar como una zona verde recreativa.

\section{AGRADECIMIENTOS}

Se agradece al Dr. Rafael Bolaños Villalobos por la información brindada para este trabajo y a la Dra. María del Carmen Bolaños directora del Área Rectora de Salud de Santo Domingo por facilitar informes relacionados con la situación de Barrio Fátima.

\section{REFERENCIAS}

Briones, F. (2010). Inundados, reubicados y olvidados: Traslado del riesgo de desastre en Motozintla, Chiapas. Revista de ingeniería, 31, 132-144.

Comisión Especial de Vivienda CEV \& Municipalidad de Santo Domingo de Heredia. (2018). Acta Número Uno, Martes o5 de Mayo 2018. Santo Domingo de Heredia: CEV. 
Comisión Nacional de Emergencias CNE. (1999). Ocasionados por Inundaciones en los cantones San Pablo y Santo Domingo de Heredia el pasado 20 de setiembre. Santo Domingo de Heredia: CNE.

Comisión Nacional de Emergencias CNE. (2008). Inspección por derrumbes y erosión lateral del río Bermúdez. Santo Domingo de Heredia: CNE.

Comisión Nacional de Emergencias CNE (2010). Informe técnico DPM-INF-0773-2010. Santo Domingo de Heredia: CNE.

Comisión Nacional de Emergencias CNE. (2016). Plan Nacional de Gestión del Riesgo 2016. San José, Costa Rica: CNE.

Comité Municipal de Emergencias de Santo Domingo de Heredia CMESD. (2010). Evento Inundaciones 26 de julio, 17:0o. Santo Domingo de Heredia: CMESD.

Delegación Policial de Santo Domingo DPSD. (2010). Informe de situación. Santo Domingo de Heredia: DPSD.

Fernández, M., Borges, J., Meléndez, G., Mora, F., Mora, J. \& Muñoz, Ch. (2013). Análisis de Gestión de Riesgo de Inundación en la ciudad de Santo Domingo de Heredia. En Adamson, M. \& Castillo, F. (Eds), Costa Rica en el tercer milenio: desafíos y propuestas para la reducción de vulnerabilidad ante los desastres (pp 266-286). San José, Costa Rica: Universidad de Costa Rica.

Macías, J. (2008). Reubicaciones por desastre. Análisis de intervención gubernamental comparada. México, D. F.: Centro de Investigaciones y Estudios Superiores en Antropología Social CIESAS.

Ministerio de Salud MS. (1999). Viviendas afectadas por inundaciones, Barrio Fátima de Heredia UPC-D-1496. San José, Costa Rica: MS.

Ministerio de Salud MS. (2007). Informe de la visita realizada a la comunidad Barrio Fátima por la emergencia en el Río Bermúdez. San José, Costa Rica: MS.

Ministerio de Salud MS. (2010). Antecedentes de la Situación de riesgo y emergencias en Barrio Fátima, San Vicente de Santo Domingo de Heredia. San José, Costa Rica: MS.

Municipalidad de Santo Domingo MSD. (2010). Informe General de la Emergencia del martes 20 de julio del 2010, acontecido en el cantón Santo Domingo, Heredia, Oficio ALM 026o-2007. Santo Domingo de Heredia: MSD.

Reyes, J., Fernández, M., Grinesky, S. \& Collins, T. (2014). Spatial Analysis of Disaster Risk in Santo Domingo de Heredia, Costa Rica, Central America. Journal of Geography and Geology, 6(3), 123-13 http://dx.doi.org/10.5539/igg.v6n3p123 